Original Article

\title{
INTER-RATER RELIABILTY OF NEONATAL INFANT PAIN SCALE (NIPS) AS A MULTIDIMENSIONAL BEHAVIORAL PAIN TOOL
}

\author{
Malarvizhi G. ${ }^{1}$, Manju Vatsa ${ }^{2}$, Roseline M. ${ }^{3}$, Nithin S. ${ }^{4}$, Sarah Paul ${ }^{5}$ \\ ${ }^{1}$ Professor,Pediatric Nursing, PSG College of Nursing, Coimbatore - 641 004, Tamilnadu, India \\ ${ }^{2,3,4}$ Principal College of Nursing AlIMS, New Delhi - 23, Assistant Professor PSGCN, Senior Resident, PSG hospitals, \\ ${ }^{5}$ Neonatologist, PSG Hospitals, Coimbatore - 641 004, Tamilnadu, India \\ Correspondence: \\ Malarvizhi G, \\ E-mail : malarvizhi2k5@yahoo.co.in
}

\begin{abstract}
:
Evaluation of pain in human neonate is complex and difficult because pain is a subjective phenomenon. Neonates cannot verbalize pain rather they express through cry and body movements. However measurement of pain provides a value of pain. There is no gold standard in pain assessment tool. JCAHO recommends selection of a valid and reliable and also age ap propriate pain assessment tool.

To determine the interobserver reliabity and clinical utility of NIPS scale To analyze the inter-rater reliability, reproducibility of NIPSscale between three observers prospective observation study was designed to establish reliability and validity of NIPS among 27 neonates who underwent venipuncture, Hepatitis vaccination (intramuscular) and heel prick at a tertiary care level III NICU. The baseline data and behavioral responsesto procedural pain were rated by three observerstrained in NIPSscale.

At the end of 100 observation across the various time intervals and three phases, inter- rater reliability of NIPS scale among the three observers were. $82, .81,75$.Acceptable psychometric properties are reported for the tool which includes cronbach's alpha levels of .9, $.85, .9$ between the observers. CNIPS was found to be highly reliable and valid multidimensional scale and practical and has good clinical utility.
\end{abstract}

Keywords: NIPS (Neonatal Infant Pain Scale), Inter-rater Reliability, Pain assessment

\section{Introduction :}

Pain is a complex phenomenon whose nature is at best elusive in neonate and it is an integral management to adults but inadequately provided to neonates ${ }^{1}$ Fifteen to $20 \%$ of neonates requires hospitalization especially in NICU due to prematurity, disease conditions, emergencies and congenital anomalies and for observation. During hospitalization newborns and infants are often exposed to numerous procedures which can be characterized as painful. Research shows the number of invasive procedures in neonates during admissions to the NICU among 54 neonates was 3283. On an average they undergo 42- 50 procedures during their stay 10, procedures a day. Among those $55 \%$ is heel stick, $26 \%$ endotracheal suctioning $15 \%$ IV cannulation., ${ }^{2,3}$ In terms of requirements for pain perception, by 20 weeks gestation, the fetal neocortex is present and pain pathways to the brain stem and thalamus are completely myelinated by 30 weeks . Pain in neonates is often under recognized and under treated in life .Neonates experience pain similarly probably more intensely than older children and adults. pain is important not only because it is an ethical expectation but also because repeated painful exposures can have deleterious consequences. ${ }^{5}$ These consequences include altered pain sensitivity (which may last into adolescence) and permanent neuroanatomic and behavioral abnormalities, as found in animal studies. ${ }^{5,6}$ It is, therefore, important for health care personnel to assess and manage pain on a regular basis in order to avoid excessive exposure ${ }^{.6}$

Optimal pain management requires competent pain assessment, which can be especially difficult to perform in neonates. The pain-assessment tool used should be multidimensional, including measurements for both physiologic and behavioral indicators of pain, because neonates cannot self-report. ${ }^{8,9}$

Assessment of pain in neonates is often challenging because neonates cannot verbalize their subjective experience. Pain behavior in neonates is exhibited as cry, 
facial expression and body movement especially to a localized pain stimulus during invasive procedures. Neonatal infant pain scale (NIPS) is scale which includes all components of behavioral responses to pain from head to foot. $^{10}$

When dealing with the assessment of pain one differentiation should be made between evaluation and measurement of painful phenomenon. ${ }^{11}$ Validity, reliability sensitivity, Internal consistency and utility and format are vital characteristics of an instrument which is designed to assess a subjective experience. ${ }^{12}$ For assessment of pain one-dimensional and multidimensional instruments are available The use of multidimensional evaluation is appropriate for individuals when self reporting is compromised which occurs among patients with cognitive /verbal deficiencies and children less than five years. Among the published multidimensional scales of assessment of pain in neonatal period, prominence is given to Neonatal infant pain scale (NIPS), premature infant pain profile (PIPPS) and CRIES. There are many gaps in the study of one-dimensional and multidimensional scales. ${ }^{13,14}$

A prospective observation study was designed to establish the interobserver reliability and clinical utility of NIPS scale among neonates exposed to invasive procedures as diagnostic and therapeutic intervention .The outcome measure of the present study was to determine the interrater reliability, reproducibility of Neonatal Infant pain Scale (NIPS) as a pain assessment tool

\section{Material and methods}

Neonatal Infant Pain Scale (NIPS; Lawrence et al., 1993)

The NIPS is a multidimensional pain scale for use in preterm infants which includes indicators for facial expression, cry, breathing patterns, arm and leg movements and state of arousal and one physiological indicator. Each indicator is given a score of 1 if present. This scale has established content, concurrent and constructs validity. ${ }^{10}$

After the approval from the ethical committee of PSGIM\&R the study was carried out in a level III tertiary care NICU,27 healthy neonates who underwent procedures such as venipunture, heel prick and immunization intramuscular (IM), (Hepatitis B) as per standard protocols were assessed for their pain responses. Neonates of 32- 40 weeks of gestation was considered to be eligible for the assessment. Babies with critical illness and cardiac defects, neonates on ventilator, with major congenital anomalies were excluded. A preliminary try out of three pain assessment tools NFCS (Neonatal Facial coding System) PIIPS (premature infan6t pain Profile) and NIPS (Neonatal Infant Pain Scale ) among 15 neonates subjected to routine venipuncture, heel prick to validate feasibility and clinical utility. NIPS was found to be more feasible.

Three observers trained in assessment of pain using NIPS scale rated the pain behavior of the neonates, before, during and after the selected procedures at time intervals of $0,15,30,60$ and 180 seconds.

Each observer had a separate NIPS instrument to observe the pain behavior of the neonate at various time intervals. A stop clock with second arm was kept at the bed side stand. It was ensured the clock was on while the neonate prepared for the procedures .Before disturbing the neonate NIPS assessment was performed by the each of observers simultaneously using individual assessment instruments. The observers rated the pain score of the neonates at three phases of the procedures such as before Osec, (prestate) during (prick/needle insertion) 15s, 30s, 60 s, and 180 seconds after the procedure.

Observer 1 was Nursing Faculty and observer 2 a senior resident doctor working in NICU and observer 3 a post graduate neonatal nurse. The observers did not talk to each other during the observation, nor were they aware about the scoring given by the other one. On completion of assessment of each neonate the nursing supervisor collected the instruments $f$ each observer and pinned together and kept aside. Each observer completed 10 assessments for each procedure which contributed to 100 observations.

\section{Statistical data analysis:}

Sample size was determined based on the estimate on $(95 \% \mathrm{Cl})$ confidence intervals of NIPS scale which indicated 
a reliability of and sensitivity values of 0.99.Using the degree of precision formula the estimated sample size was 27 and number of observations required were 100 . With regard to the statistical correlation the agreement between the observers the interclass correlation coefficient and its statistical significance was used. The correlation was considered to be excellent when the value of coefficient was greater than 0.75 , good when the coefficient ranged from 0.75- 0.40 and poor when correlation ranged below.40 it was considered poor and $p<$ $0.01^{12}$

Inter-rater reliability of NIPS scale among the three observers was determined by Pearson's correlation and the internal consistency of NIPS was evaluated using cronbach's $\alpha$.

\section{Results :}

Among 27 neonates enrolled in the study, there were 15 males (55.5\%) and 12 female neonates (44.4\%) with the mean age of 2 days (range 1-28 days) and the mean gestational age was 37weeks (SD 2.1 weeks) and with the mean body weight of $2.248 \mathrm{gms}$ (SD $448 \mathrm{gms}$ ).

\section{Discussion :}

The advantage of using NIPS to assess the neonate's behavioral responses to procedural pain was that it did not require the additional assessment skills or equipments. Although numerous neonatal pain assessment scales are available to assess and evaluate acute pain the composite multidimensional responses can be assessed through NIPS as it includes the total body response of a neonate to a localized pain stimulus including the behavioral parameter and state of alertness.

Amar M Taskande, and colleagues ${ }^{15}$ assessed 80 neonates responses to venipuncture .The mean NIPS score during the procedural was $(5.74-6.41 \mathrm{p}<0.001)$ higher in two groups compared to the baseline $(1.84,1.56 \mathrm{P}<0.001) \mathrm{A}$ significant increase is NIPS score was recorded in the present study $1.68,1.81,1.61$ between three raters before the procedure among the raters $P<0.001$ and during the procedure $(4.41,4.20,5.29 p<0.001)$. Also consistent with a similar study done at NICU at Iran with mean NIPS score of
1.99,5. 62 and 3.59 at various phases of procedures.$^{16} \mathrm{~A}$ high level of inter-rater reliability was found between the three raters $r=.82, .75$ and .81 in the present study. Suraseranivongse and colleagues ${ }^{17}$ also reported that on comparison of three pain scales CRIES, CHIPPS and NIPS among 22 post operative frenulectomy neonates the most reliable and practical tool was NIPS with a high interclass correlation of 0.98 at $95 \% \mathrm{Cl}$.

Lawrence J A Clock and associates ${ }^{10}$ videotaped 38 neonates responses on 90 observations using NIPS at various phases of the procedure such as before during and after each intrusive procedure there was a significant difference overtime with high correlations which ranged from .92 to .97 across successive minutes of observation. The six component scores of NIPS had high internal consistency and the cronbach's $\alpha$ were $.95, .87$ and .88 . These study findings are consistent and similar to the above with 27 subjects and 100 observations among three raters cronbach's $\alpha$ were .90 .90 and .86 before during and after the procedure despite the heterogeneity of procedures. Amir and colleagues ${ }^{18}$ also reported similar results on assessing tongue tie post operative pain.

The present study has some limitations. As the raters directly observed and rated the neonate's behavioral responses to pain they could not compare using another pain scale simultaneously. Rather during various procedures PIPPS and NFCS was used and NIPS was found to be very practical hence the reliability was tested. While using PIPPS a pulse oximeter was needed to monitor the heart rate and oxygen saturation and attaching the probe caused false recordings while the neonate was pricked which led to vigorous leg movement which could be elicited through NIPS. But from an evolutionary perspective, behavioral indicators are specifically designed to elicit care giving .NFCS includes facial actions only, in case of sick neonates facial actions may be dampened.

This study has much strength since NIPS is a multidimensional scale the total responses of neonate could be measured including the state of alertness. Concerning practicality NIPS was found to be more 
NIPS score:

\begin{tabular}{|c|c|c|c|c|c|c|c|}
\hline S.NO & Observers & $0 \mathrm{sec}$ & $15 \mathrm{sec}$ & $30 \mathrm{sec}$ & $60 \mathrm{sec}$ & $180 \mathrm{sec}$ & overall \\
\hline 1. & $1 \& 2$ & 1.68 & 4.41 & 4.22 & 3.90 & 4.71 & 3.51 \\
\hline 2. & $1 \& 3$ & 1.81 & 4.20 & 3.75 & 3.55 & 3.92 & 3.24 \\
\hline 3. & $3 \& 2$ & 1.61 & 5.29 & 4.31 & 3.79 & 4.35 & 3.56 \\
\hline
\end{tabular}

(Table 1 ) describes the mean NIPS score among the three observers $P \varangle 0.001$

All the values are at $p<0.001$.The overall values indicates the mean score of NIPS at 100 observation at 0 sec before procedure, $15 \mathrm{sec}-60 \mathrm{Sat}$ during the procedures, and $180 \mathrm{sec}$ as end of the procedure. The behavioral responses to procedural pain are as before the procedure there was no pain, during the procedural phase $>4$ indicate severe pain and end of procedure $>3$ indicate moderate pain.

Inter - rater reliability of NIPS: The study demonstrates high inter-rater reliability of this scale. Pearson's correlations ranged from .71 to .96 across successive minutes of observations. The scale had inter - class correlation $>.90$.

\begin{tabular}{|c|c|c|c|c|c|c|c|}
\hline S.NO & Observers & Osec & $15 \mathrm{sec}$ & $30 \mathrm{sec}$ & $60 \mathrm{sec}$ & $180 \mathrm{sec}$ & \\
\hline 1. & $1 \& 2$ & .85 & .78 & .83 & .85 & .96 & \\
\hline 2. & $1 \& 3$ & .81 & .82 & .73 & .81 & .71 & \\
\hline 3. & $3 \& 2$ & .76 & .76 & .82 & .86 & .83 & \\
\hline
\end{tabular}

Table 2- shows the inter-rater reliability of NIPS at various time intervals among the three observes. $P<0.001$

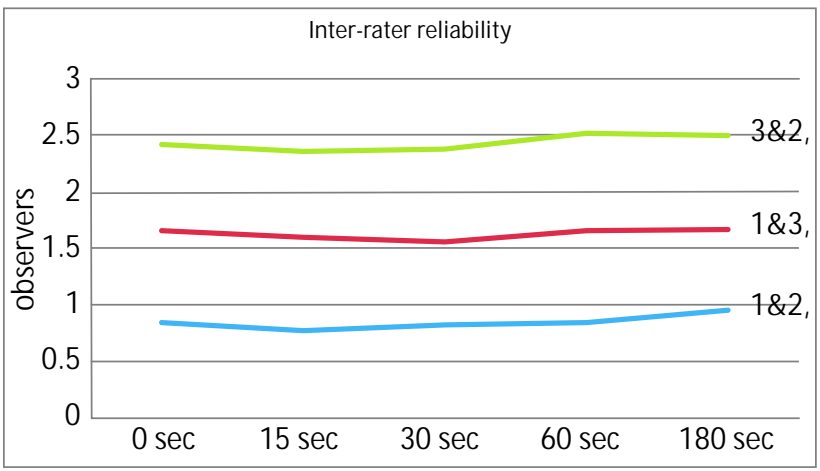

Figure 1 - Inter-rater- reliability at various phases of procedure between three observers

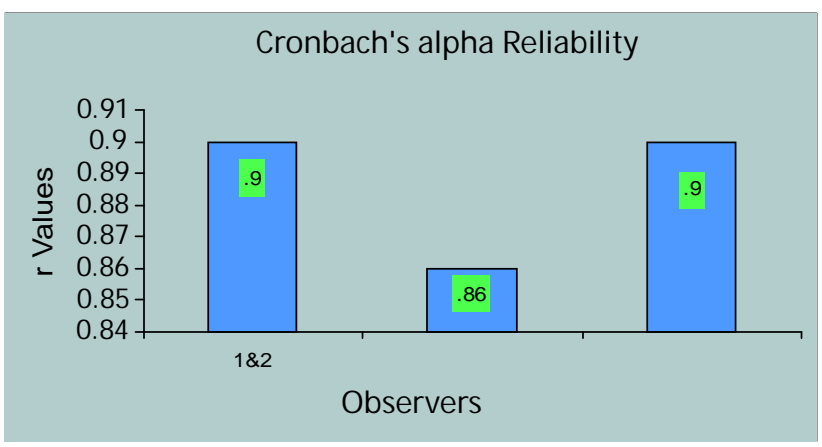

Fig-2 Overall internal consistency\& reliability of NIPS on 100 observationsamong the rater

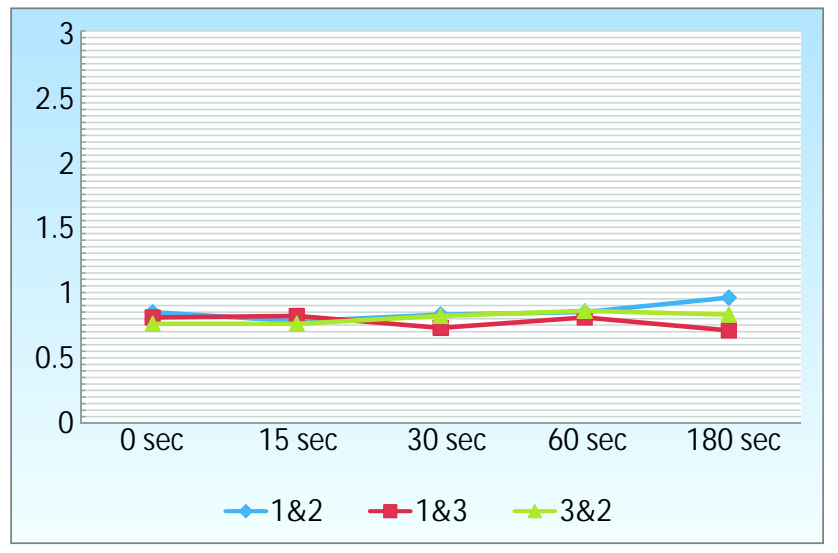

Figure 2 -Inter-rater- reliability at various phases of procedure between three observers

Internal consistency: Evaluation of internal consistency of NIPS scale among the three raters was analyzed by coefficient of Alpha (cronbach's $\alpha$ ).The values depicted in the bar graph shows there is very high internal consistency among the three observers at various phases of the procedure. 
satisfactory because of its ease and feasibility .In terms of clinical utility it can be used across all settings as it does not require additional monitors to record saturations and heart rate. Accurate assessment is essential for managing neonatal pain .Tools have been developed specifically for the purpose of facilitating assessment,which results in appropriate intervention. NIPS was successfully implemented to assess pain in neonate as fifth vital sign in southern California hospital through education and to its ease of application in clinical practice..$^{19}$ In this study also NIPS is found to have high clinical utility.

\section{References :}

1. Anand KJS, the International Evidence-Based Group for Neonatal Pain. Consensus statement for the prevention and management of pain in the newborn. Arch Paediatr Adolesc Med. 2001; 155: 173-18

2. Simons $S H$, vanDijk $M$, Anand KS, Roofthooft $D$, van Lingen $R A$, Tibboel D Do we still hurt newborn babies? A prospective study of procedural pain and analgesia in neonates. Arch Pediatr Adolesc Med. 2003 157(11):1058.

3. Brenda C and Zeev N. Kain. Procedural Pain in Neonates: The New Millennium. Pediatrics 2005; 115; 1073.

4. Anand KJS, Hickey PR. Pain and its effects in the human neonate and fetus. New Engl J Med. 1987; 317(21):1321-28,

5. American Academy of Pediatrics, Committee on Fetus and Newborn. Prevention and management of pain and stress in the neonate. Pediatrics. 2000; 105:454-61.

6. Johnston CC, Stevens. Experience in a neonatal intensive care unit affects pain response. Pediatrics. 1996; 98 (5):925-30.

7. Ruda MA, Ling QD, Hohmann AG, Peng YB, Tachibana T. Altered nociceptive neuronal circuits after neonatal peripheral inflammation. Science. 2000; 289:628-31

8. Taddio A, Shah V, Gilbert-MacLeod C, Katz J. Conditioning and hyperalgesia in newborns exposed to repeated heel lances. JAMA. 2002; 288:857-61

9. Oberlander TF, Grunau RE, Whitfield MF, Fitzgerald C, Pitfield S, Saul JP. Biobehavioral pain responses in former extremely low birth weight infants at four months corrected age. Pediatrics. 2000; 105(1). Available at www.pediatrics.org/Cgi/content/full/105/1/e6.

\section{Conclusion :}

Pain assessment is a preliminary step in pain management in neonate and accurate assessment of neonatal pain using appropriate scale sets the stage for effective pain management strategies. The outcome measures to of NIPS pain are found to be a reliable, valid and practical tool with high significance to clinical friendliness. NIPS is a suitable multidimensional instrument to assess neonatal pain.

\section{Acknowledgements :}

We acknowledge the nurses of PSG hospitals NICU for their cooperation during the study.

10. Lawrence J, AclockD, Kay J, Macmurray SB, Duilberg C. The developmental tool to assess neonatal pain. Neonatal Netw.1993; 12(6):59-66

11. Guinsberg R, BrancoMf, PeresCDA, Shinzato AR, Kapleman BI.Reliabilty of two behavioral tools to assess pain in preterm neonates. Sao Paulo Med J: 2003;12(2):72-76.

12. Polit DF, Beck CT.Nursing Research. $9^{\text {th }}$ ed.Wolters kluwer: Lippincot; 2011

13. KrechelSW, BlinderJ., CRIES: a new post operative pain measurement score. Initial testing of validity and reliability. Pediatr Anaesth: 1995;5(1):53-61.

14. DJ Garry, E Swoboda, A Elimian and R Figueroa; A video study of pain relief during newborn male circumcision. J Perinat: 2006; 26, 106-110.

15. Amar M. Taksande KY, Vilhekar, Jain M, ChitreD, Pain Response of Neonates to Venipuncture.Indian J Pediatr: 2005; 72 (9): 751-53.

16. SaranghiF,Mollahadi M, Ebadi A ,Matinzadeh ZH,Tadrisi SD.Validity and reliability of Neonatal Infant Pain Scale in NICU in Iran. Pak J Med Sci.2011;27(5):1087-91.

17. Suraseranivongse S., Kaosaard R., Intakong P., Pornsiriprasert S., Karnchana Y., Kaopinpruck J., Sangjeen K., A comparison of postoperative pain scales in neonates. Br J Anaesth: 2006; 97(4) 540-45.

18. Amir LH, James JP, Beatty J. Review of tongue-tie release at tertiary maternity hospital. J Pediatric Child Health 2005; 41:243-5.

19. Gallo AM. The Fifth Vital Sign: Implementation of the Neonatal Infant Pain Scale. JOGNN: 2003; 32, ( 2 ):191-205 